\title{
Short-Term Clinical Follow-Up After Thrombolytic Therapy in Patients With Prosthetic Valve Thrombosis: A Single-Center Experience
}

\author{
Akshyaya Pradhan ${ }^{\mathrm{a}, \mathrm{b}}$, Monika Bhandaria, Vikas Gupta ${ }^{\mathrm{a}}$, Pravesh Vishwakarma ${ }^{\mathrm{a}}$, Rishi Sethi ${ }^{\mathrm{a}}$, \\ Varun Shankar Naraina, Gaurav Chaudharya, Sharad Chandra ${ }^{a}$, Sudhanshu Dwivedi ${ }^{a}$
}

\begin{abstract}
Background: Thrombolytic therapy has evolved as an alternative to surgery for prosthetic valve thrombosis (PVT). Therefore, this retrospective, single-center study aimed to evaluate the clinical profile of PVT and the role of thrombolytic therapy in patients with PVT.

Methods: Data from a total of 16 consecutive patients with PVT enrolled between January 2017 and January 2018 at a tertiary care center in India were retrospectively evaluated. PVT was diagnosed based on clinical presentation, transthoracic echocardiography, and fluoroscopy. All patients received 0.25 MU intravenous (IV) bolus streptokinase over $30 \mathrm{~min}$, followed by a $0.1 \mathrm{MU} / \mathrm{h}$ IV infusion for a maximum of $72 \mathrm{~h}$. Transthoracic echocardiography and fluoroscopy were repeated after completion of thrombolysis session. The clinical endpoints were death and hemodynamic success within $24 \mathrm{~h}$ of thrombolytic therapy or during the hospital stay, and major complications, including stroke or major bleeding (intracranial bleed or bleeding requiring transfusion or surgical treatment) during the hospital stay and within 3 months of thrombolytic therapy.
\end{abstract}

Results: The median age of the patients was $40 \pm 11.60$ years and about $62.5 \%(n=10)$ were females. The median time between the valve placement and presentation for PVT was 3 years (range: 1 4 years). The peak gradient across the thrombotic mitral and aortic valve was $43.79 \pm 18.47$ and $93.5 \pm 33.11 \mathrm{mmHg}$, respectively. At 3 days post-thrombolysis, peak gradient across both mitral valve (15.91 \pm 7.56 ; mean gradient: $8.45 \pm 4.01)$ and aortic valve $(23.50 \pm 6.45$; mean gradient: $13.60 \pm 3.83)$ decreased significantly $(\mathrm{P} \leq 0.05)$. The thrombolytic therapy was successful in $13(81.25 \%)$ patients. While, two $(12.50 \%)$ patients died, none developed stroke or myocardial infarction during the study period.

Conclusions: The present study with short-term follow-up dem-

Manuscript submitted August 2, 2019, accepted August 28, 2019

aDepartment of Cardiology, King George's Medical University, Shah Mina Road, Lucknow, Uttar Pradesh 226003, India

${ }^{b}$ Corresponding Author: Akshyaya Pradhan, Department of Cardiology, King George's Medical University, Lucknow 226007, India.

Email: akshyaya33@gmail.com

doi: https://doi.org/10.14740/cr924 onstrated the acceptable clinical efficacy of thrombolytic therapy. However, larger trials with a greater number of patients and longer follow-up are warranted to establish the safety and effectiveness of thrombolytic therapy in patients with PVT.

Keywords: Prosthetic valve thrombosis; Thrombolytic therapy; Streptokinase

\section{Introduction}

Prosthetic valve thrombosis (PVT) is a life-threatening complication after valve replacement and depends mainly on the: 1) valve design (the risk of PVT is higher with caged-ball type valves vs. bileaflet, tilting disc valves); 2) structure (PVT risk is higher for mechanical vs. bioprosthetic valves); and 3) location (risk of PVT is higher in mitral vs. aortic position) [1]. The incidence of PVT is 1 - 2 per patient-year, despite improvements in the design of prosthetic heart valves, and the use of anticoagulant therapy and better surgical techniques $[2,3]$. The 11-year probability of PVT in patients with bioprosthetic or mechanical valves on the left-side (mitral or aortic position) is $0.01 \%$ to $0.02 \%$ [4]. Left-sided PVT is rare than right-sided PVT in developed countries, but its prevalence is $6.1 \%$ within 6 months of valve replacement in India [5].

Treatment options for left-sided PVT mainly include surgery, thrombolysis, anticoagulant therapy and antiplatelet therapy, depending upon the prosthesis location, thrombus size and clinical status [1]. Surgery is the first-line of treatment for obstructive left-sided PVT. However, reported intraoperative mortality in critically-ill patients is as high as $50 \%$ $[6,7]$. Thrombolytic therapy (TT) is an alternative to surgical treatment, which can be used in patients with stable clinical condition and small thrombus $[1,6,8]$. However, TT may be associated with a high risk of emboli formation and hence, is reserved for high-risk surgical candidates $[9,10]$. In low-risk patients, the use of TT remains controversial.

We report a single-center experience of TT with streptokinase in a small cohort of patients with PVT who underwent primary aortic valve replacement or mitral valve replacement at a tertiary care center. We retrospectively evaluated the short- 
term outcomes of this patient cohort.

\section{Patients and Methods}

Data was retrospectively collected from patients with PVT who underwent primary aortic valve or mitral valve replacement at a tertiary care center in India between January 2017 and January 2018. The study protocol was approved by the institutional ethics committee at the study center. This study was conducted in compliance with the ethical standards of the responsible institution on human subjects as well as with the Helsinki Declaration. The aortic or mitral valve implanted in the patients were mechanical valves (St. Jude Medical prosthesis valves (SJM-25, 29, 27, 31) and ATS prosthesis valves). The diagnosis of PVT was based on clinical presentation, transthoracic echocardiography (TTE) and fluoroscopy. Patients complaining of dyspnoea, chest pain, syncope, or palpitation; showing a high-pressure gradient across the prosthetic valve on TTE; and reduced or fixed leaflet mobility on fluoroscopy were considered to have PVT and were included in the study. Patients with endocarditis, non-obstructive thrombosis or patients with a prosthesis mismatch (characterized by high gradients on TTE but normal leaflet motion on fluoroscopy) were excluded from the study. Informed consent was obtained from all the enrolled patients. All patients received 0.25 MU intravenous (IV) bolus streptokinase over $30 \mathrm{~min}$, followed by a $0.1 \mathrm{MU} / \mathrm{h}$ IV infusion for a maximum of $72 \mathrm{~h}$. TTE and fluoroscopy were repeated after the completion of thrombolysis session. After successful thrombolysis, patients were restarted on acenocoumarol/warfarin (anticoagulant therapy) according to the prothrombin time international normalized ratio (INR). Patients with no or subtherapeutic response after TT were advised valve replacement therapy. The patients were closely followed up clinically and telephonically for 3 months after TT.

\section{Endpoints and definitions}

The clinical outcomes were death or absence of hemodynamic response, and hemodynamic success within $24 \mathrm{~h}$ of TT and major complications including stroke or major bleeding (intracranial bleed or bleeding requiring transfusion or surgical treatment) during the hospital stay or within 3 months of TT. Death or absence of hemodynamic response was considered as failure of TT, whereas normalization of transvalvular gradients irrespective of leaflet mobility was considered as hemodynamic success [11].

\section{Statistical analysis}

Statistical analysis was performed using SPSS version 16.0. Data was expressed as mean \pm standard deviation (SD) or frequency (percentage). Paired $t$-test or Wilcoxon-Rank test was used to analyse statistical significance. A P value of $<0.05$ was considered as significant.
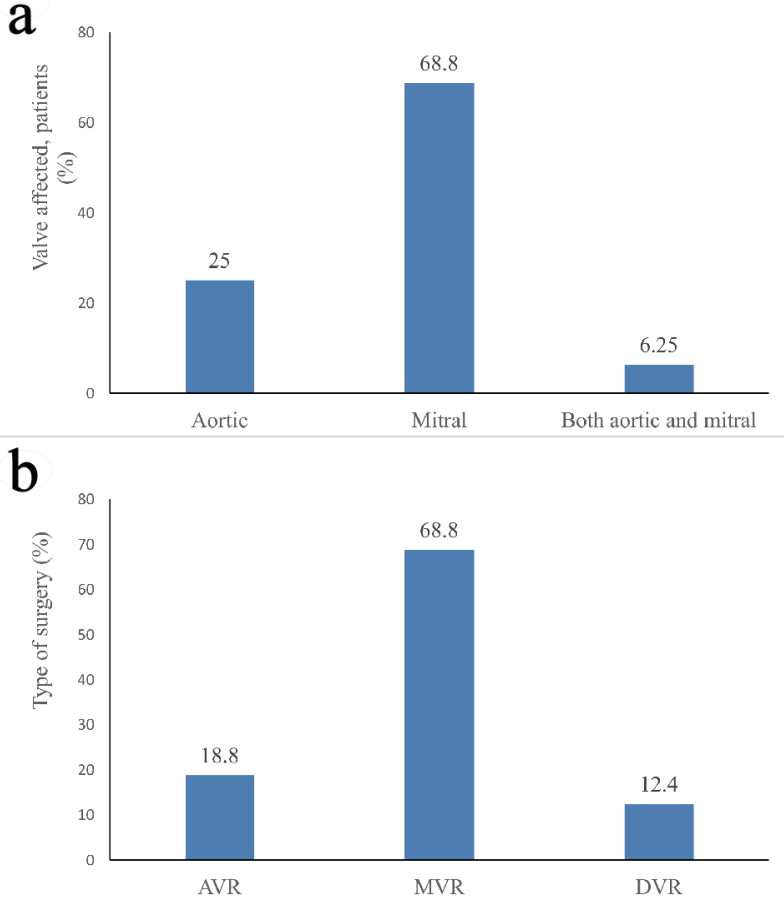

Figure 1. Details of: (a) type of affected valve; (b) type of surgery. AVR: aortic valve replacement; MVR: mitral valve replacement; DVR: double valve replacement.

\section{Results}

\section{Baseline characteristics}

A total of 16 patients with PVT were enrolled with mean age of $40 \pm 11.60$ years; $10(62.5 \%)$ were females. About $68.8 \%$ of patients underwent mitral valve replacement. The distribution of the affected valves and the type of surgery are shown in Figure 1. The median time between the valve placement and presentation for PVT was 3 years (range: 1 - 4 years). The symptoms at presentation were mainly dyspnoea (15, $93.75 \%$ ), followed by chest pain, syncope, and palpitation. While one $(6.25 \%)$ patient was in New York Heart Association (NYHA) class III, seven (43.75\%) were in NYHA class I and four $(25.00 \%)$ were in NYHA class II. All patients were on acenocoumarol/warfarin (anticoagulation therapy). Subtherapeutic international normalized ratio (INR $<1.6$ ) was found in $14(87.5 \%)$ patients, out of which two patients stopped taking drugs 2 days before admission, while one had stopped drugs due to rectal bleeding. Other baseline characteristics are shown in Table 1.

\section{Study outcomes}

The peak gradient across the thrombotic mitral valve was 43.79 $\pm 18.47 \mathrm{mmHg}$ (mean gradient: $24.06 \pm 8.76$ ) whereas the peak gradient across the thrombotic aortic valve was $93.5 \pm 33.11$ $\mathrm{mmHg}$ (mean gradient: $57.50 \pm 26.71$ ). Three days after throm- 
Table 1. Baseline and Demographic Characteristics of the Study Population

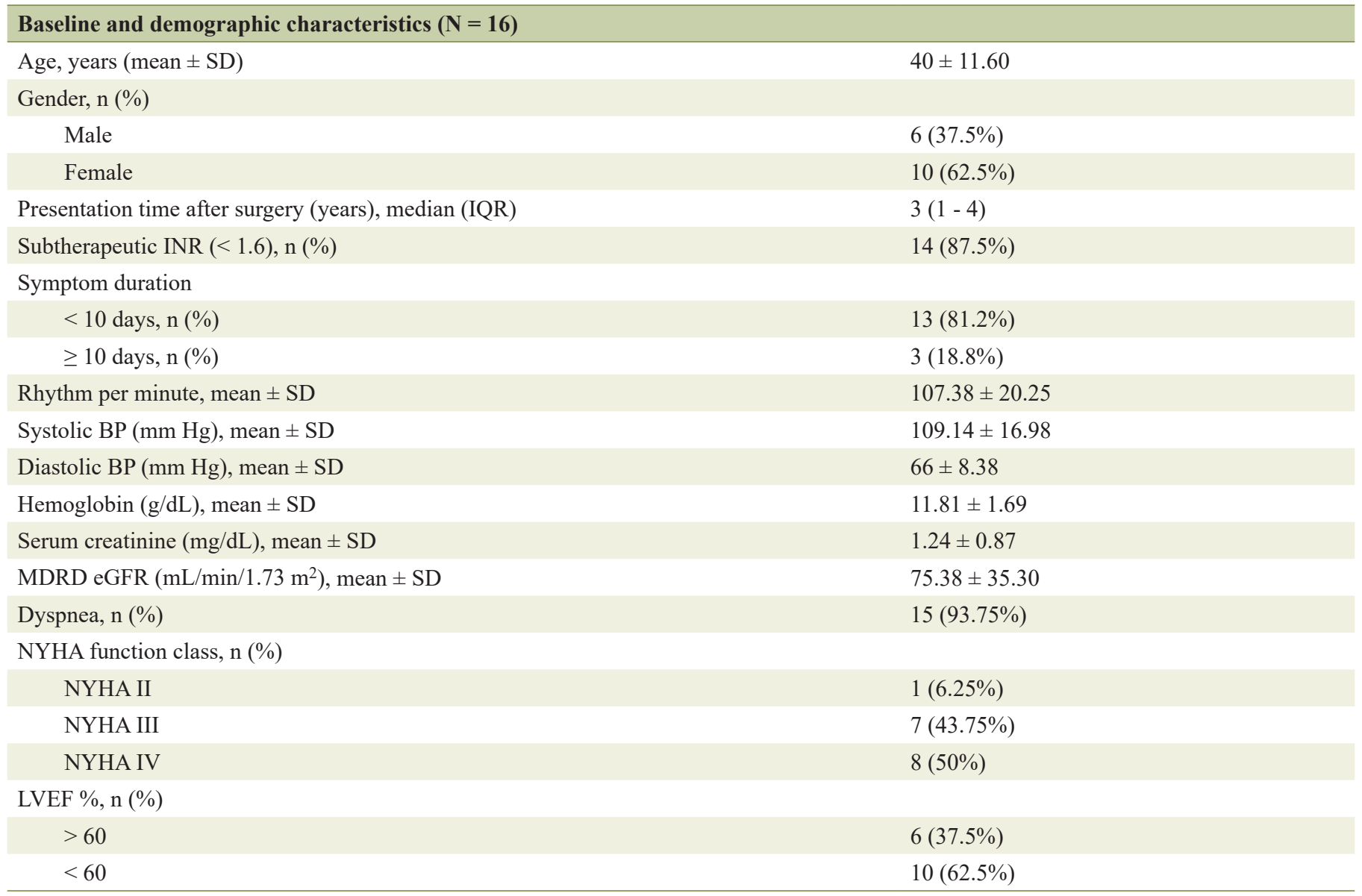

IQR: interquartile range; BP: blood pressure; LVEF: left ventricular ejection fraction; MDRD: modification of diet in renal disease; eGFR: estimated glomerular filtration rate; NYHA: New York Heart Association; AVR: aortic valve replacement; MVR: mitral valve replacement; DVR: double valve replacement; SD: standard deviation; INR: international normalized ratio.

bolysis, the peak gradient across both mitral valve $(15.91 \pm 7.56$; mean gradient: $8.45 \pm 4.01)$ and aortic valve $(23.50 \pm 6.45$; mean gradient: $13.60 \pm 3.83)$ decreased significantly as compared to the peak gradient prior to TT $(\mathrm{P} \leq 0.05)$. The TT was hemodynamically successful in $13(81.25 \%)$ patients. Over the 3 months period, two $(12.50 \%)$ patients died. None of the patients suf- fered from a stroke or myocardial infarction (Table 2).

\section{Discussion}

The current guidelines have emphasized on early diagnosis and

\section{Table 2. Clinical Outcomes}

\begin{tabular}{|c|c|c|c|}
\hline Transvalvular gradient & Pre-thrombolysis & 3 days post-thrombolysis & $P$ value \\
\hline \multicolumn{4}{|l|}{ Mitral valve } \\
\hline Peak gradient mean $\pm \mathrm{SD}$ & $43.79 \pm 18.47$ & $15.91 \pm 7.56$ & $<0.05$ \\
\hline \multicolumn{4}{|l|}{ Aortic valve } \\
\hline Peak gradient mean \pm SD & $93.5 \pm 33.11$ & $23.50 \pm 6.45$ & $<0.05$ \\
\hline Hemodynamic success & $13(81.25 \%)$ & & \\
\hline
\end{tabular}

SD: standard deviation. 
urgent treatment of patients with PVT with either TT or surgery. Low surgical risk, NYHA functional class III/IV, contraindication to fibrinolysis, recurrent valve thrombosis, possible or suspected pannus, large clot $\left(>1.0 \mathrm{~cm}^{2}\right)$, mobile thrombus $>$ $0.3 \mathrm{~cm}$ in diameter, presence of left atrial thrombus, absence of need of coronary artery bypass graft surgery (CABG) for concomitant coronary artery disease and availability of expert surgical team favour surgical treatment for PVT. On the contrary, factors which favour TT include: 1) high surgical risk; 2) NYHA I/II functional class; 3) first episode of PVT; 4) presence of mass consistent with thrombus; 5) presence of a small clot $\left(<1 \mathrm{~cm}^{2}\right)$ without any contraindication for surgery; 6$)$ absence of immediate surgical expertise; and 7) need for cardiac surgery for other indications (e.g. CABG). For non-obstructive left-sided PVT and stable obstructive PVT, medical stabilization with diuretics and optimization of anticoagulation with unfractionated heparin (UFH) followed by vitamin K antagonists and aspirin is recommended. For right-sided PVT, either optimization of anticoagulation therapy or TT, depending upon clinical condition may be suitable [12]. In resource-limited settings like India, considering the high cost and high intraoperative mortality of surgery for PVT, and unavailability of surgical expertise at all centers and at all time points [12], TT may be preferred over surgery, especially in centers without onsite surgical backup.

The current study confirms the clinical effectiveness of streptokinase as TT in patients with PVT in terms of high hemodynamic success and less mortality. Among the enrolled patients, all had left-sided PVT with maximum (68.8\%) cases of mitral valve thrombosis. Further, majority $(68.75 \%)$ of the patients were in the NYHA class I and II, $87.5 \%$ patients had subtherapeutic anticoagulation (INR $<1.6$ in 14 patients) and showed a significantly decreased transvalvular gradient $(\mathrm{P}<$ 0.05 ) after TT. Few studies have reported $69-75 \%$ rate of inadequate anticoagulation and 2 - 3 times more prevalence of mitral valve vs. aortic valve thrombosis $[13,14]$.

A systematic review of trials of TT in PVT patients, reported that the success rate of TT ranged from $58 \%$ to $90 \%$, the 30 day mortality rate ranged from $0.8 \%$ to $16.7 \%$, the rate of thromboembolism ranged from $1.7 \%$ to $17.6 \%$, and major bleeding ranged from $1.7 \%$ to $12 \%$ [15]. Another review which included Indian studies with sample size ranging from 10 to 119 reported a success rate of $59-100 \%$ [16]. In the current study, the sample size was 16 with observed success rate of $81.25 \%$ and mortality of $12.50 \%$ over 3 -month follow-up. There were no complications of stroke or myocardial infarction. The high success rate may be due to the inclusion of less critical patients in the study, small sample size and prolonged duration of treatment (up to 72 h). A study conducted by Karthikeyan et al [17] reported a success rate of only 59\% in NYHA class III/IV patients. Other studies have reported that TT is not efficacious in restoring the valve function in patients with recurrent PVT $[18,19]$. In contrast, the meta-analysis conducted by Karthikeyan et al [20] which included 690 patients with PVT, concluded that emergency surgery is non-superior than TT in restoring the valve functions.

\section{Study limitations}

The current study has few limitations. First, it is a single-center experience with small sample size. Second, long-term followup was not conducted in the study.

\section{Conclusions}

In the present study, TT was hemodynamically successful with low short-term mortality in patients with left-sided PVT (majorly mitral valve thrombosis). Long-term studies in larger patient cohorts are needed to further establish the clinical efficacy and safety of TT in patients with PVT.

\section{Acknowledgments}

None to declare.

\section{Financial Disclosure}

None to declare.

\section{Conflict of Interest}

The authors have none to declare.

\section{Informed Consent}

It is a retrospective evaluation of records; hence no inform consent taken from patients.

\section{Author Contributions}

$\mathrm{AP}$ and $\mathrm{MB}$ were instrumental for the concept and design of the study. VG and PV did the data acquisition and data analysis. AP and MB did the statistical analysis as well as the literature search. VG, GC and PV prepared the manuscript. RS, SC, $\mathrm{SD}$ and $\mathrm{VN}$ reviewed and edited the manuscript. AP drafted the final version of the manuscript.

\section{References}

1. Roudaut R, Serri K, Lafitte S. Thrombosis of prosthetic heart valves: diagnosis and therapeutic considerations. Heart. 2007;93(1):137-142.

2. Cannegieter SC, Rosendaal FR, Briet E. Thromboembolic and bleeding complications in patients with mechanical heart valve prostheses. Circulation. 1994;89(2):635-641.

3. Akins CW. Results with mechanical cardiac valvular prostheses. Ann Thorac Surg. 1995;60(6):1836-1844.

4. Hammermeister KE, Sethi GK, Henderson WG, Oprian C, Kim T, Rahimtoola S. A comparison of outcomes in men 11 years after heart-valve replacement with a mechanical valve or bioprosthesis. Veterans Affairs Coop- 
erative Study on Valvular Heart Disease. N Engl J Med. 1993;328(18):1289-1296.

5. Talwar S, Kapoor CK, Velayoudam D, Kumar AS. Anticoagulation protocol and early prosthetic valve thrombosis. Indian Heart J. 2004;56(3):225-228.

6. Roudaut R, Labbe T, Lorient-Roudaut MF, Gosse P, Baudet E, Fontan F, Besse P, et al. Mechanical cardiac valve thrombosis. Is fibrinolysis justified? Circulation. 1992;86(5 Suppl):II8-15.

7. Sato N, Miura M, Itoh T, Ohmi M, Haneda K, Mohri H, Nitta S, et al. Sound spectral analysis of prosthetic valvular clicks for diagnosis of thrombosed Bjork-Shiley tilting standard disc valve prostheses. J Thorac Cardiovasc Surg. 1993;105(2):313-320.

8. Ledain LD, Ohayon JP, Colle JP, Lorient-Roudaut FM, Roudaut RP, Besse PM. Acute thrombotic obstruction with disc valve prostheses: diagnostic considerations and fibrinolytic treatment. J Am Coll Cardiol. 1986;7(4):743751.

9. Birdi I, Angelini GD, Bryan AJ. Thrombolytic therapy for left sided prosthetic heart valve thrombosis. J Heart Valve Dis. 1995;4(2):154-159.

10. Gagnon RM, Beaudet R, Lemire J, Carignan L, Guimond C, Cousineau G. Streptokinase thrombolysis of a chronically thrombosed mitral prosthetic valve. Cathet Cardiovasc Diagn. 1984;10(1):5-10.

11. Nawale JM, Chaurasia AS, Nalawade DD, Abdagire N. Clinical profile of patients with prosthetic valve thrombosis treated with fibrinolysis. Journal of the Practice of Cardiovascular Sciences. 2018;4(2):109.

12. Nishimura RA, Otto CM, Bonow RO, Carabello BA, Erwin JP, 3rd, Fleisher LA, Jneid H, et al. 2017 AHA/ACC Focused Update of the 2014 AHA/ACC Guideline for the management of patients with valvular heart disease: a report of the American College of Cardiology/American Heart Association Task Force on clinical practice guide- lines. Circulation. 2017;135(25):e1159-e1195.

13. Caceres-Loriga FM, Perez-Lopez H, Morlans-Hernandez K, Facundo-Sanchez H, Santos-Gracia J, Valiente-Mustelier J, Rodiles-Aldana F, et al. Thrombolysis as first choice therapy in prosthetic heart valve thrombosis. A study of 68 patients. J Thromb Thrombolysis. 2006;21(2):185190.

14. Hirachan A, Roka M, Prajapati D, Adhikari CM, Bishal KC, Khadka MB, Maskey A, et al. Prosthetic valve thrombosis in a tertiary cardiac centre. Nepalese Heart Journal. 2017;14(1):9-11.

15. Lim WY, Lloyd G, Bhattacharyya S. Mechanical and surgical bioprosthetic valve thrombosis. Heart. 2017;103(24):1934-1941.

16. Caceres-Loriga FM, Morais H. Thrombotic obstruction in left-side prosthetic valves: Role of thrombolytic therapy. Indian Heart J. 2015;67(Suppl 2):S10-12.

17. Karthikeyan G, Math RS, Mathew N, Shankar B, Kalaivani M, Singh S, Bahl VK, et al. Accelerated infusion of streptokinase for the treatment of left-sided prosthetic valve thrombosis: a randomized controlled trial. Circulation. 2009;120(12):1108-1114.

18. Balasundaram RP, Karthikeyan G, Kothari SS, Talwar KK, Venugopal P. Fibrinolytic treatment for recurrent left sided prosthetic valve thrombosis. Heart. 2005;91(6):821822 .

19. Kumar BM, Paul GJ, Swaminathan N, Venkatesan S, Hussain GZ, Gnanavelu G. Assessment of hemodynamic and clinical response in thrombolytic therapy for prosthetic valve thrombosis. Indian Heart J. 2017;69:S6.

20. Karthikeyan G, Senguttuvan NB, Joseph J, Devasenapathy N, Bahl VK, Airan B. Urgent surgery compared with fibrinolytic therapy for the treatment of left-sided prosthetic heart valve thrombosis: a systematic review and meta-analysis of observational studies. Eur Heart J. 2013;34(21):1557-1566. 